\title{
Hormonal response to physical exercise
}

\author{
Codruta Lencu*, Teodora Alexescu**,Monica Lencu ${ }^{* * *}$, Carmen Georgescu $^{*}$ \\ * Department of Endocrinology, University of Medicine Cluj-Napoca, Romania \\ ** 4th Medical Clinic, University of Medicine Cluj-Napoca, Romani \\ *** Medical Centrum"Interservisan"Cluj-Napoca, Romania
}

\section{Hypothesis}

Hormonal response to physical exercise is a field of wide interest, which depends on the nature, intensity and duration of exercise. The effects of aerobic/anaerobic physical exercise on the endocrine system were monitored.

\section{Material and method}

\section{Material}

$* 60$ young subjects (aged between 20-35 years)

* without associated chronic diseases

* with normal weight

* not under drugs treatment

* non smokers

* who followed a constant physical exercise program over the past year

Material
group A (30) performed anaerobic exercise for 50 minutes:
running on the treadmill at a speed of 8
$\mathrm{~km} /$ hour ( 20 minutes)
walking on the treadmill at a speed of $5 \mathrm{~km} / \mathrm{hour}$
(10 minutes)
running on the treadmill at a speed of 8
$\mathrm{~km} /$ hour ( 20 minutes)
group B $(30)$ performed aerobic exercise for 50 minutes:
running on the treadmill at a speed of 15
$\mathrm{~km} /$ hour (20 minutes)
- walking on the treadmill at a speed of $5 \mathrm{~km} / \mathrm{hour}$
$(10$ minutes)
running on the treadmill at a speed of
$15 \mathrm{~km} / \mathrm{hour}(20$ minutes)

Method

All the studied subjects underwent the measurement of $\mathrm{GH}, \mathrm{PRL}$ cortisolemia, adrenaline, noradrenaline, insulinemia, estradiol, before exercise, at the end of exercise at 1 hour, 6 hours and 24 hours after exercise.

\section{Results}

Changes in levels of hormones for group A (values are means)

\begin{tabular}{|c|c|c|c|c|c|}
\hline Hormone & Pre-test & $\begin{array}{c}\text { Immediately } \\
\text { post-test }\end{array}$ & $1 \mathrm{~h}$ post-test & $6 \mathrm{~h}$ post-test & $\begin{array}{c}24 \text { h post- } \\
\text { test }\end{array}$ \\
\hline GH & $1,3 \mathrm{ng} / \mathrm{ml}$ & $28,1 \mathrm{ng} / \mathrm{ml}_{* \star}$ & $28,0 \mathrm{ng} / \mathrm{ml}_{* \star}$ & $9,7 \mathrm{ng} / \mathrm{ml}_{*}$ & $2,5 \mathrm{ng} / \mathrm{ml}$ \\
\hline PRL & $10 \mathrm{ng} / \mathrm{ml}$ & $33,8 \mathrm{ng} / \mathrm{ml}_{\star \star *}$ & $11,9 \mathrm{ng} / \mathrm{ml}$ & $11,0 \mathrm{ng} / \mathrm{ml}$ & $10,8 \mathrm{ng} / \mathrm{ml}$ \\
\hline Cortisol & $220 \mathrm{nmol} / \mathrm{l}$ & $571 \mathrm{nmol} / I_{*}$ & $550 \mathrm{nmol} / \mathrm{I}_{*}$ & $377 \mathrm{nmol} / \mathrm{l}$ & $220 \mathrm{nmol} / \mathrm{l}$ \\
\hline Adrenaline & $70 \mathrm{pg} / \mathrm{ml}$ & $140 \mathrm{pg} / \mathrm{ml}_{* \star}$ & $140 \mathrm{pg} / \mathrm{ml}_{* *}$ & $110 \mathrm{pg} / \mathrm{ml}$ & $98 \mathrm{pg} / \mathrm{ml}$ \\
\hline Noradrenaline & $102 \mathrm{pg} / \mathrm{ml}$ & $244 \mathrm{pg} / \mathrm{ml}_{* *}$ & $240 \mathrm{pg} / \mathrm{ml}_{* *}$ & $201 \mathrm{pg} / \mathrm{ml}$ & $191 \mathrm{pg} / \mathrm{ml}$ \\
\hline Insuline & $11 \mu \mathrm{u} / \mathrm{ml}$ & $7,1 \mu \mathrm{u} / \mathrm{ml}_{*}$ & $7,1 \mu \mathrm{u} / \mathrm{ml}_{*}$ & $7,8 \mu \mathrm{u} / \mathrm{ml}_{*}$ & $10 \mu \mathrm{u} / \mathrm{ml}$ \\
\hline Estradiol & $34,8 \mathrm{pg} / \mathrm{ml}$ & $58,4 \mathrm{pg} / \mathrm{ml}_{* *}$ & $54,1 \mathrm{pg} / \mathrm{ml}_{* *}$ & $50,8 \mathrm{pg} / \mathrm{ml}_{* *}$ & $47,1 \mathrm{pg} / \mathrm{ml}_{* *}$ \\
\hline
\end{tabular}

Changes in levels of hormones for group B (values are means)

\begin{tabular}{|c|c|c|c|c|c|}
\hline Hormone & Pre-test & $\begin{array}{c}\text { Immediately } \\
\text { post-test }\end{array}$ & $1 \mathrm{~h}$ post-test & $6 \mathrm{~h}$ post-test & $\begin{array}{c}24 \mathrm{~h} \text { post- } \\
\text { test }\end{array}$ \\
\hline GH & $1,7 \mathrm{ng} / \mathrm{ml}$ & $20,1 \mathrm{ng} / \mathrm{ml} *$ & $20,1 \mathrm{ng} /\left.\mathrm{m}\right|_{* *}$ & $10,8 \mathrm{ng} / \mathrm{ml}_{*}$ & $1,9 \mathrm{ng} / \mathrm{ml}$ \\
\hline PRL & $12 \mathrm{ng} / \mathrm{ml}$ & $36,3 \mathrm{ng} / \mathrm{ml} *$ & $12,7 \mathrm{ng} / \mathrm{ml}$ & $12,5 \mathrm{ng} / \mathrm{ml}$ & $12,1 \mathrm{ng} / \mathrm{ml}$ \\
\hline Cortisol & $181 \mathrm{nmol} / \mathrm{l}$ & $648 \mathrm{nmol} / \mathrm{I}_{*}$ & $571 \mathrm{nmol} / /_{*}$ & 450nmol/l & $188 \mathrm{nmol} / \mathrm{l}$ \\
\hline Adrenaline & $71 \mathrm{pg} / \mathrm{ml}$ & $139 \mathrm{pg} / \mathrm{ml} \mathrm{l}_{* *}$ & $139 \mathrm{pg} / \mathrm{ml}_{\mathrm{k*}}$ & $119 \mathrm{pg} / \mathrm{ml}$ & $89 \mathrm{pg} / \mathrm{ml}$ \\
\hline Noradrenaline & $102 \mathrm{pg} / \mathrm{ml}$ & $250 \mathrm{pg} /\left.\mathrm{ml}\right|_{* *}$ & $250 \mathrm{pg} / \mathrm{ml} \mathrm{l}_{* *}$ & $202 \mathrm{pg} / \mathrm{ml}$ & $185 \mathrm{pg} / \mathrm{ml}$ \\
\hline Insuline & $14 \mu \mathrm{u} / \mathrm{ml}$ & $8 \mu \mathrm{u} / \mathrm{ml}_{*}$ & $8 \mu \mathrm{u} / \mathrm{ml}_{*}$ & $9,7 \mu \mathrm{u} / \mathrm{ml}$ & $10,7 \mu \mathrm{u} / \mathrm{ml}$ \\
\hline Estradiol & $40,6 \mathrm{pg} / \mathrm{ml}$ & $65 \mathrm{pg} /\left.\mathrm{m}\right|_{* *}$ & $64,1 \mathrm{pg} /\left.\mathrm{m}\right|_{* *}$ & $61,7 \mathrm{pg} /\left.\mathrm{ml}\right|_{* *}$ & $60,7 \mathrm{pg} /\left.\mathrm{m}\right|_{* *}$ \\
\hline
\end{tabular}

\section{Conclusions}

$>$ During physical activity, a significant increase of GH in both groups occurred; the values remained high at one hour after exercise $>\mathrm{PRL}$ increased during physical training proportionally to the difficulty of training in all the studied women, and returned to its initial value after the cessation of training.

$>$ Cortisol varied proportionally to the intensity of training

$>$ Physical exercise determined an increase of catecholamine secretion; the response was more marked for noradrenaline.

$>$ During physical activity, we found a significant decrease of insulin in both groups of women.

$>$ Estradiol secretion increased significantly in both groups of women; the values remained high at 24hours after cessation of physical training.

$>$ The imbalance between energy consumption and the much higher energy expenditure causes the onset of neuroendocrine mechanisms of adaptation of the body to increased physical exercise. 